\title{
Extermination Speed of an Imidacloprid and Flumethrin Polymer Matrix Collar against Larvae, Nymphs and Adults of Haemaphysalis longicornis
}

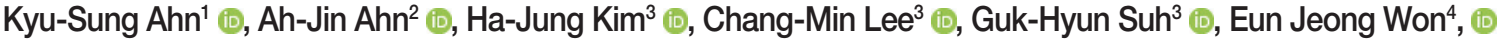 \\ SungShik Shin ${ }^{1, *}$ (i) \\ 'Department of Parasitology, College of Veterinary Medicine, Chonnam National University, Gwangju 61186, Korea; ${ }^{2}$ Gwangju Metropolitan \\ Government Research Institute of Public Health and Environment, Gwangju 61954, Korea; ${ }^{3}$ Department of Internal Medicine, College of Veterinary \\ Medicine, Chonnam National University, Gwangju 61186, Korea; ${ }^{4}$ Department of Parasitology and Tropical Medicine, Chonnam National University \\ Medical School, Hwasun-gun 58128, Korea
}

\begin{abstract}
The objective of this study was to evaluate the efficacy of an imidacloprid $10 \%$ and flumethrin $4.5 \%$ polymer matrix collar against the developmental stages of Haemaphysalis longicornis infesting dogs using the hair from treated dogs in a semi-in-vitro assay set. When incubated with $0.5 \mathrm{~g}$ of the hair collected from the dogs installed with the drugembedded collar after 10 days, average death rate of the larval, nymphal, and adult $H$. longicornis was $21.5 \%, 77.9 \%$, and $100 \%$ at $30 \mathrm{~min}, 1 \mathrm{hr}$, and $2 \mathrm{hr}$, respectively. This study showed the larval stages as well as the nymphal and adult stages of $H$. longicornis ticks are killed upon contact with the hair from dogs treated with the collar within $2 \mathrm{hr}$.
\end{abstract}

Key words: Haemaphysalis Iongicornis, Seresto, tick, imidacloprid, flumethrin, polymer, collar

\section{INTRODUCTION}

The speed and ability to kill both the juvenile and adult ticks are important factors for an acaricide to successfully prevent the transmission of tick-borne diseases in dogs. The tickborne pathogens are transmitted from ticks to animals or to humans during a tick sucks blood and injects saliva containing pathogens into the host tissues. The transmission of diseases such as Lyme borreliosis takes 24 to $48 \mathrm{hr}$ after a tick bite [1]. The Powassan virus causing encephalitis in humans can be transmitted to host animals in 15 min of tick attachment [2]. The transmission course emphasizes the importance of the speed kill of acaricides against ticks both for the prevention against tick bites and tick-borne pathogens. The ability of an acaricide to kill the larval stage of ticks as well as the nymphal and adult stages is also important, because evidence for the transovarial transmission of pathogens via the female-egg-larvae route in ticks have been well documented. Examples of

- Received 18 July 2021, revised 11 October 2021, accepted 12 October 2021.

*Corresponding author (sungshik@jnu.ac.kr)

(c) 2021. Korean Society for Parasitology and Tropical Medicine

This is an Open Access article distributed under the terms of the Creative Commons Attribution Non-Commercial License (https://creativecommons.org/licenses/by-nc/4.0) which permits unrestricted non-commercial use, distribution, and reproduction in any

medium, provided the original work is properly cited. transovarial transmission include Borrelia miyamotoi by Ixodes scapularis tick, Leishmania infantum by Rhipicephalus sanguineus, Rickettsia bellii by Ixodes loricatus and Babesia equi by Haemaphysalis longicornis [3-8]. In dogs, the transmission of pathogens via the transovarial route from infected female ticks to dogs by larval ticks was documented on Babesia gibsoni by Haemaphysalis hystrics and Rickettsia rickettsia by Rhipicephalus sanguineus [5, $6]$.

The role of larval ticks should therefore be considered important in the transmission of tick-borne diseases. It should be ensured that an acaricide kills larvae as well as ticks in the nymph and adult stages. However, it is difficult to assess the in-vivo clinical efficacy of drugs against the larval stage of ticks on host animals due to their minute size which hinders them from being found on the host's skin, especially in the thick and/or colored hairs. For this reason, larvicidal assays of acaricides have used in-vitro systems such as Food and Agriculture Organization larval packet test (LPT) or larval immersion microassay (LIM) $[9,10]$. However, limitations exist with these assays to obtain information on in-vivo acaricidal efficacy on the host animal skin after acaricides are applied.

The host hair from dogs treated with certain insecticides such as pyrethroids or neonicotinoids are coated with a small amount of the chemicals, which is enough to kill ticks or fleas 
upon contact with the hair [11-13]. It was reported that adult cat fleas (Ctenocephalides felis Bouche' 1835) died within 50-90 min on contact with hair from imidacloprid (Advantage ${ }^{\circledR}$, Elanco Animal Health, Indianapolis, Indiana, USA)-treated dogs, whereas adult fleas, having contact with hair of untreated control dogs, were found alive even 7 days after the beginning of the experiments [11]. The hair removed from a treated dog with a contact-kill acaricide can, therefore, be used to assess the speed of kill against ticks. Seresto ${ }^{\circledR}$ (Elanco Animal Health) is a contact-kill collar product for dogs and cats that provides long term broad spectrum parasiticidal activity by combining the insecticidal properties of imidacloprid (10\%) with the acaricidal properties of flumethrin (4.5\%). The active ingredients spread from the site of direct contact over the entire skin surface of the treated animal and provide the long term steady and continuous release of the ingredients by the collar matrix system [14]. The product offers a breakthrough in protection against fleas and ticks for dogs. It was documented that a preventive efficacy against ticks in dogs varied between $91.2 \%$ and $100 \%$ (overall mean: $94.7 \%$ ) for 8 months [15].

The purpose of this study is twofold: to assess the time taken to exterminate larvae, nymphs, and adults of the species in contact with the hair from the host dogs treated with the collar using a semi-in-vitro assay, and to evaluate the efficacy of $10 \%$ imidacloprid and $4.5 \%$ flumethrin polymer matrix collar against Haemaphysalis longicornis tick infestation on dogs.

\section{MATERIALS AND METHODS}

\section{Ethics approval}

A randomized, controlled efficacy trial was conducted in the animal care facility at the College of Veterinary Medicine, Chonnam National University, Gwangju, Korea. The design and experimental procedures used in this study followed the rules and regulations of the Korea Animal Protection Law (No. 13023, articles 8 and 9). This study was approved by the Institutional Animal Care and Use Committee of Chonnam National University (CNU IACUC-YB-2018-81).

\section{Animals}

Before the semi-in-vitro assay using the hair from treated dogs was performed, an in-vivo clinical efficacy of an ectoparasiticide against $H$. longicornis infestation in dogs was conducted to confirm its efficacy in field conditions. Twelve healthy young dogs between 2 and 6 months old of both sexes with no history of tick infestation or exposure to tick habitats were enrolled in this study. Each dog received 3 doses of a vaccine against canine distemper, adenovirus Type 2, parvovirus, and parainfluenza (Vanguard ${ }^{\circledR}$ Plus 5, Zoetis, Parsippany, New Jersey, USA). All dogs were dewormed with a combination of praziquantel, pyrantel pamoate, and fenbantel (Drontal ${ }^{\circledR}$ plus; Elanco Animal Health) before being enrolled in this study. Dogs were individually housed in wire mesh cages $(90 \times 70 \times$ $60 \mathrm{~cm}$ ) and were fed commercial feed once per day, with water provided ad libitum. Six dogs were randomly assigned to the treatment group and 6 dogs in the untreated control group. On day -3, a polymer matrix collar containing 10\% imidacloprid and 4.5\% flumethrin (Seresto ${ }^{\circledR}$, Elanco Animal Health) was applied to all 6 dogs in the treatment group according to the manufacturer's instructions: dogs $\leq 8 \mathrm{~kg}$ received a small collar and dogs $>8 \mathrm{~kg}$ received a large collar.

\section{In vivo drug efficacy evaluation}

On day 0 , all 12 dogs were naturally exposed to ticks by walking them for $1 \mathrm{hr}$ in wooded and bushy areas with tall grass and leaf litter in Yeongkwang-gun, Jeonnam, Korea $\left(35^{\circ} 14^{\prime} 39.5^{\prime \prime} \mathrm{N} 126^{\circ} 35^{\prime} 08.7^{\prime \prime} \mathrm{E}\right)$ where H. longicornis was found to be the dominant species of ticks in a preliminary investigation. The exposure of dogs to ticks was repeated on day 30. From day 1 , each dog was clinically examined, and ticks were searched for once daily until all ticks became detached from the host. Dogs were examined daily for the presence of ticks by thumb counting, with examination of the cephalic, ventral, dorsal, and leg regions. Both nymphal and adult stages of ticks attached to the skin of the host animal were counted, and those ticks crawling around on the skin were not counted. Representative tick specimens ( 1 or 2 adult ticks per dog) were identified as H. longicornis based on morphology [16].

The efficacy of the collar against ticks was calculated as follows: Efficacy $=$ (mean total number of ticks on control dogsmean total number of ticks on treated dogs)/(mean total number of ticks on control dogs) $\times 100$. Collars were deemed effective against ticks if the calculated efficacy, based on arithmetic and geometric means, was at least 90\% [17].

\section{In vitro drug efficacy evaluation}

For the semi-in-vitro assay, the larval, nymphal, and adult stages of ticks were co-cultured with hair from dogs collected on day 10 after the application of the collar containing $10 \%$ 
imidacloprid and 4.5\% flumethrin. Hair was collected from 3 dogs randomly selected from the treatment group and 3 dogs from the untreated control group at the dorsal medial line of each dog approximately $20 \mathrm{~cm}$ away from the collar. Collected hair was individually weighed, cut into pieces of approximately 5-10 mm long, and $0.5 \mathrm{~g}$ of hair from each dog was placed in a $50 \mathrm{ml}$ conical centrifuge tube (Hyundai Micro Co. Ltd., Seoul, Korea). Each conical centrifuge tube contained a $1.5 \mathrm{ml}$ micro-centrifuge tube sealed at the inner top wall of the $50 \mathrm{ml}$ tube using a hot melt glue gun (Fig. 1). A small amount of sterilized cotton soaked with distilled water was inserted into the micro-centrifuge tube to provide humidity inside the $50 \mathrm{ml}$ tube. Disposable gloves and scissors were used to prevent any contamination between hair samples.

Fifteen larvae, nymphs, or adults of $H$. longicornis were placed inside each of the $50 \mathrm{ml}$ tube containing $0.5 \mathrm{~g}$ of the host hair collected from dogs installed with the collar or from the untreated control dogs. Larvae of $H$. longicornis were obtained from newly hatched eggs from engorged adult females in the laboratory, and additional nymphs and adults were collected from wooded fields at the study site for natural infection. The tubes were closed tightly and were incubated for 0.5, 1, 2, 4, 6, and $24 \mathrm{hr}$ at room temperature $\left(25^{\circ} \mathrm{C}\right)$. At each incubation time point, the number of live and dead ticks were counted using a dissecting microscope. Ticks were considered dead if they showed no movement and did not move or respond to prodding with a fine-tipped needle. Acaricidal efficacy was determined by comparing the mean number of live ticks remaining on the treated dog hair with the mean number of dead mites remaining on the control dog hair.

\section{RESULTS}

\section{Efficacy of $10 \%$ imidacloprid and $4.5 \%$ flumethrin in a polymer matrix collar against $H$. longicornis ticks in a field trial}

In the untreated control group of 6 dogs on day 0 to $\mathrm{H}$. longicornis-populated fields 3 days after the application of the polymer matrix collar containing 10\% imidacloprid and 4.5\% flumethrin, an average of 30.0, 38.1, and 36.1 ticks were found on the host animals on days 1, 2, and 3 after exposure to ticks (Data not shown in Table). The highest number of ticks attached to the host skin were observed on day 2 because there were some ticks still crawling around on day 1 . The majority of ticks finished feeding on blood and detached from the host from day 4 after exposure, leaving an average of 6.6 ticks remaining on the host skin on day 4 and 0.3 ticks on day 7 . Ticks were not found on the host skin from day 8 onwards in the control group. On the other hand, no ticks were found on any of the 6 dogs applied with the collar during the study period.

Although the average number of ticks attached to dogs was smaller than the first exposure, an average of 18.7, 21.2, and 14.9 ticks were found on the host animals on days 1, 2, and 3 after exposure to ticks (Data not shown in Table). The majority of ticks finished feeding on blood and detached from the host from day 4 after exposure, leaving an average of 4.6 ticks remaining on the host skin on day 4 and 0.4 ticks on day 7 .
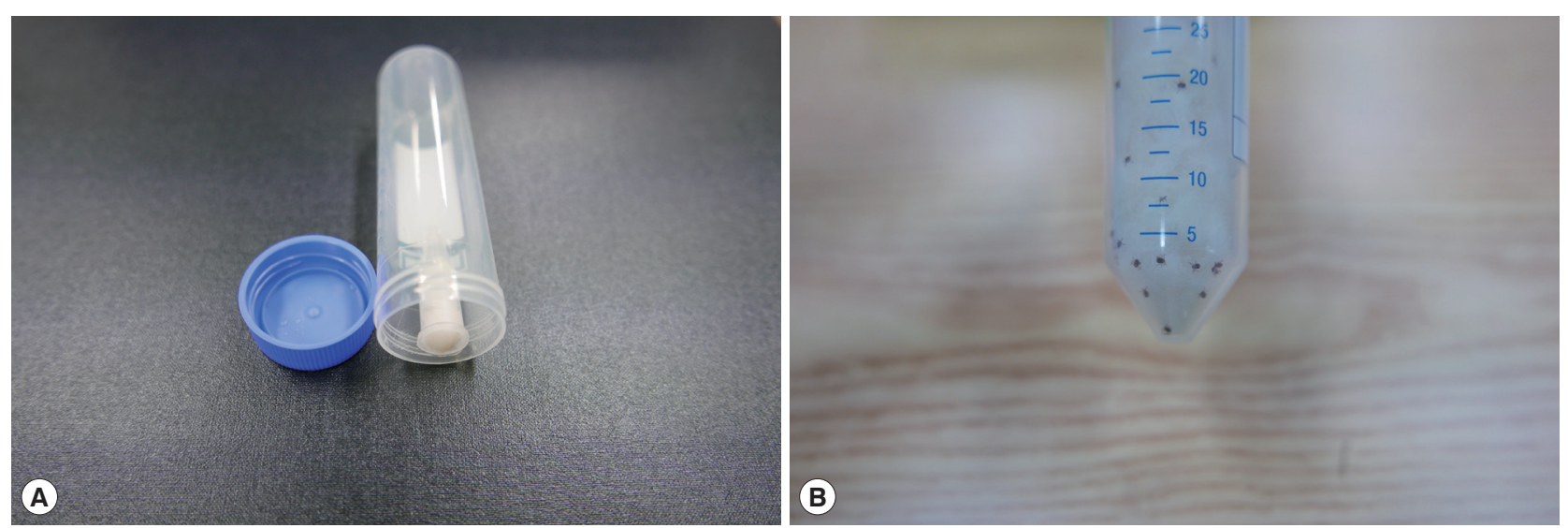

Fig. 1. A semi-in vitro acaricide assay set. (A) A disposable $50 \mathrm{ml}$ conical centrifuge tube with a $1.5 \mathrm{ml}$ micro-centrifuge tube sealed to inner top wall using a hot melt glue gun. A small amount of sterilized cotton soaked with distilled water was inserted into the micro-centrifuge tube to provide humidity in the $50 \mathrm{ml}$ tube. (B) The dog hairs and 15 Haemaphysalis longicornis ticks (black dots) were co-incubated in the $50 \mathrm{ml}$ conical tube. 
Same as in the first exposure, ticks were not found from any of the 6 dogs treated with the drug-embedded collar on the second exposure. Dogs in both groups did not show any abnormal clinical signs of side-effects such as loss of appetite, vomiting, depression, or lethargy during the study period.

\section{Efficacy of $10 \%$ imidacloprid and $4.5 \%$ flumethrin in a polymer matrix collar against the developmental stages of ticks by the semi-in-vitro assay}

The efficacy of host hair collected from dogs treated with the polymer matrix collar containing 10\% imidacloprid and 4.5\% flumethrin against larval-, nymphal- and adult-stage ticks is summarized in Table 1 . When larval stage ticks of $H$. longicornis ticks were incubated with the host hair from the drug-treated dogs, 24.4\%, 95.5\%, and 100.0\% of ticks were killed within $30 \mathrm{~min}, 1 \mathrm{hr}$, and $2 \mathrm{hr}$ after incubation respectively. Similar results were obtained when the nymphal- and adult-stages of $H$. longicornis were incubated with the host hair. Overall, averages of $21.5 \%, 77.9 \%$, and $100 \%$ of ticks were killed within 30 $\min , 1 \mathrm{hr}$, and $2 \mathrm{hr}$ after incubation with host hair from dogs in the drug-treated group respectively. On the contrary, all developmental stages of $H$. longicornis ticks incubated with the host hair from the untreated control dogs survived throughout the test period ( $24 \mathrm{hr}$ ).

\section{DISCUSSION}

Some chemicals such as pyrethrins, phrethroids, neonicotinoids, phenylpyrazoles, metaflumizone, and macrocyclic lactones of the spinosyn group are contact-kill insecticides [13]. Flumethrin, a second generation of photostable pyrethrins, and imidacloprid, one of neonicotinoid insecticides are the 2 active ingredients embedded in the polymer matrix collar that kill fleas and ticks on contact. The semi-in-vitro assay used in this study shows that the larval, nymph, and adult stages of $H$. longicornis ticks are efficiently killed upon contact with hair from dogs treated with the collar within $2 \mathrm{hr}$.

It is difficult to assess the time to kill ticks that are on the host animal down to the hourly or minute intervals in conventional clinical studies of drug efficacy using live animals because it takes considerable, and challenging, time to find live ticks that are scattered around the host body after infestation. It is especially so when measuring the time to kill nymphs or larvae due to their minute sizes that are difficult to find in the midst of the host's hair. The in-vitro evaluation of a drug's efficacy using either a filter paper impregnated with a test drug or immersion of ticks in a drug-diluted solution before incubation in filter paper clips was therefore frequently used $[18,19]$. However, the filter paper or immersion tests do not represent the speed of the killing by an acaricide in situ on the host animal's skin. The semi-in-vitro assay using the hair from drug-treated dogs, on the other hand, can assess the efficacy of the speed of extermination by an acaricide down to hourly or even minute intervals.

The results of our study also indicate that tick-borne diseases by $H$. longicornis ticks can be further prevented by a polymer matrix collar containing 10\% imidacloprid and $4.5 \%$ flumethrin, because ticks are expected to be killed even before they attach themselves to the host's skin and start feeding on blood. The Asian long-horned tick (or bush tick in Australia), H. longicornis, is prevalent in Asian countries like China, Japan, Korea, and the far-east of Russia; and it has also been found in Australia, New Zealand, several Pacific islands (Caledonia, Fiji, Western Samoa, Tonga, Vanuatu), and more recently in North America [20-23]. Besides its role as a vector for Theileria orientalis in livestock and severe fever with thrombocytopenia syndrome virus (SFTSV) in humans $[24,25]$, this species is the vector of Babesia gibsoni for dogs in Asia [26], and is also a putative vector of Hepatozoon canis [27], Rickettsia japonica [28], Borrelia, Bartonella, Anaplasma, and Ehrlichia [29].

Both pyrethroids and neonicotinoids act through contact. Pyrethroids such as flumethrin, permethrin, and deltamethrin kill insects and mites by opening $\mathrm{Na}+$ channels, inducing nerve cell membrane depolarization [13]. Neonicotinoids such as imidacloprid, nitenpyram, and dinotefuran act like agonists on postsynaptic nicotinic acetylcholine receptors, mainly in motoneurons, inducing nerve membrane depolarization, which causes spastic paralysis in insects. The absence of ticks on the host's skins in the treatment group in the field exposure study is therefore due to the contact killing of imidacloprid and flumethrin embedded in the collar, even before ticks inserted their hypostomes into the hosts' skins.

Although the number of dogs enrolled in this study is small to draw any statistical significance, this study clearly illustrates that the drug-treated collar (Seresto ${ }^{\circledR}$ ) can successfully prevent dogs from undergoing H. longicornis infestation, as shown by the high efficacy against tick infestation in the 2 field exposures of dogs to areas where H. longicornis is populated (Data not shown in Table). Number of previous reports demonstrated the long-term efficacy of the product against ticks and fleas 


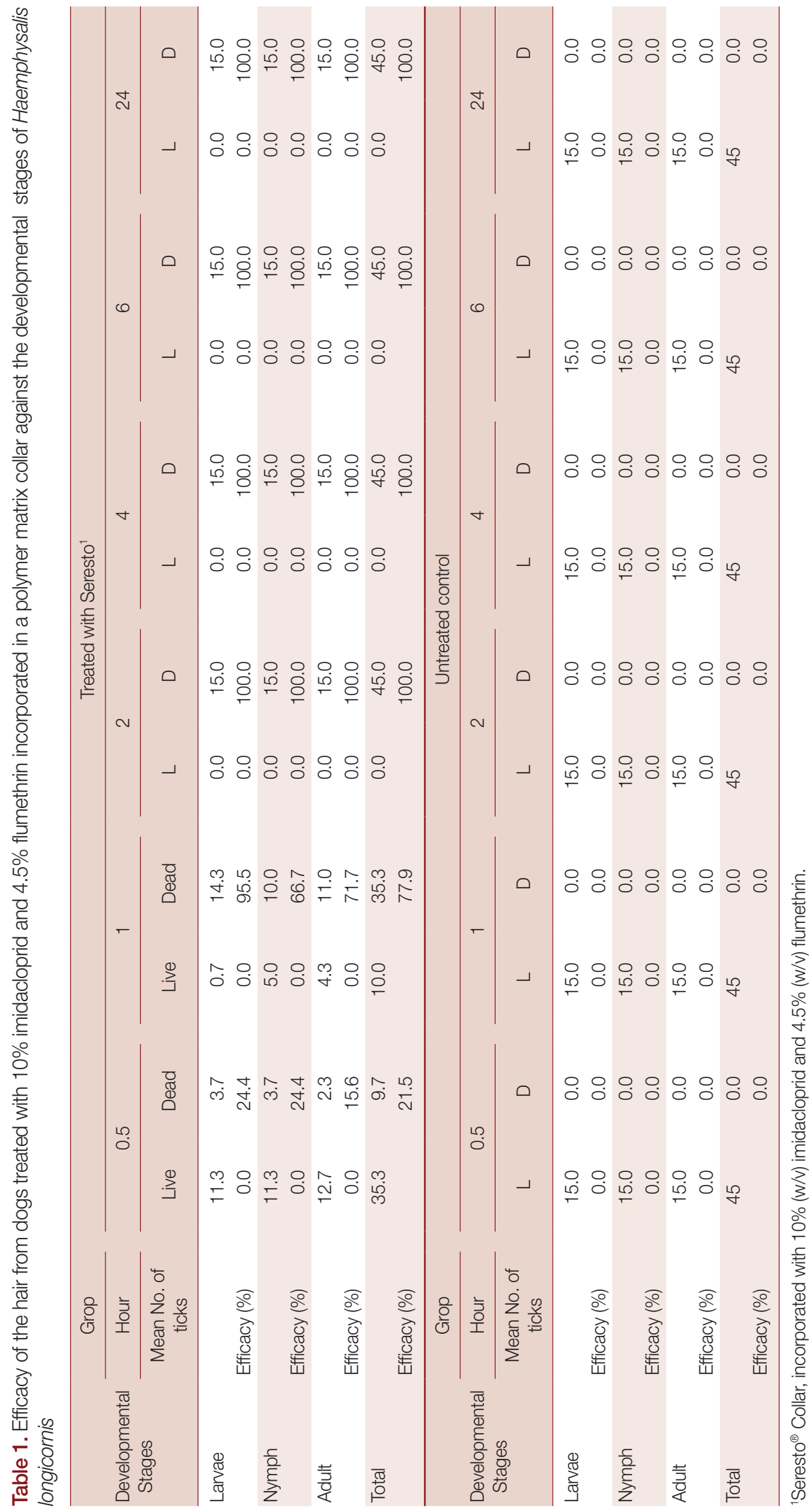


in dogs and cats. In multicenter clinical field studies in dogs and cats in Europe, Stanneck et al. [15] reported that the imidacloprid/flumethrin collar proved to reduce tick counts by at least $90 \%$ and flea counts by at least $95 \%$ for a period of at least 7-8 months in cats and dogs under field conditions. Brianti et al. [30] also reported the efficacy of the collar in 82 dogs that the mean intensity of ticks (Rhipicephalus sanguineus sensu alto) decreased to 3.5 with a reduction of $92.5 \%$. In a parallel group study on the speed of kill, repellent ability, and acaricidal efficacy of Seresto ${ }^{\circledR}$ and Broadline ${ }^{\circledR}$ (Merial) against artificially-induced infestations with Ixodes ricinus on cats, Seresto ${ }^{\circledR}$ proved to be $100 \%$ effective against adult $I$. ricinus at all assessment times $(6,12,24$, and $48 \mathrm{hr}$ after infestation) throughout the month-long study [30]. Since the tick counts were done only on days 0 and 30 and only 12 dogs were enrolled in our study, a direct comparison of results with previous studies would not be relevant, and the study group (only 6 treated dogs and 6 as a control) is too small for a reliable statistical analysis. However, the combination of the field study using 12 dogs and the semi-in-vitro assay using the host hair from treated dogs demonstrates the excellent efficacy of the polymer matrix collar containing $10 \%$ imidacloprid and $4.5 \%$ flumethrin against all 3 developmental stages of $H$. longicornis ticks.

The semi-in-vitro assay using the hair from treated dogs may not be applicable to evaluate acaricides with systemic modes of action. While some active ingredients that are used in spoton formulations or collars that act upon contact with the arthropods; others are systemic and/or mixed in their mode of action with a transcutaneous penetration and a plasma circulation [13]. Systemic insecticides are drugs whose active ingredient is distributed by blood circulation regardless of the route of administration. Systemic insecticides currently being used in dogs against tick infestation were identified which include imidacloprid, spinosad, fluralaner, afoxolaner, sarolaner, lufenuron, and nitenpyram [31-33].

In conclusion,this study showed that ticks are successfully prevented by applying the polymer matrix collar containing $10 \%$ imidacloprid and $4.5 \%$ flumethrin. and the semi-in-vitro assay using the hair from dogs can be a useful tool to evaluate the contact-kill efficacy of acaricides.

\section{ACKNOWLEDGMENT}

This study was supported in part by the Ministry of Agricul- ture, Food and Rural Affairs, Republic of Korea (Grant No. 315016-3-C00).

\section{CONFLICT OF INTEREST}

The authors declare that they have no competing interests.

\section{REFERENCES}

1. Piesman J, Mather TN, Sinsky R, Spielman A. Duration of tick attachment and Borrelia burgdorferi transmission. J Clin Microbiol 1987; 25: 557-558. https://doi.org/10.1128/jcm.25.3.557-558.1987

2. Ebel GD, Kramer LD. Short Report: duration of tick attachment required for transmission of powassan virus by deer ticks. Am J Trop Med Hyg 2004; 71: 268-271. https://doi.org/10.4269/ajtmh.2004.71.3.0700268

3. Rollend L, Fish D, Childs JE. Transovarial transmission of Borrelia spirochetes by Ixodes scapularis: a summary of the literature and recent observations. Ticks Tick-borne Dis 2013; 4: 46-51. https://doi.org/10.1016/j.ttbdis.2012.06.008

4. Ikadai H, Sasaki M, Ishida H, Matsuu A, Igarashi I, Fujisaki K, Oyamada T. Molecular evidence of Babesia equi transmission in Haemaphysalis longicornis. Am J Trop Med Hyg 2007; 76: 694697. https://doi.org/10.4269/ajtmh.2007.76.694

5. Jongejan F, Su BL, Yang HJ, Berger L, Bevers J, Liu PC, Fang JC, Cheng YW, Kraakman C, Plaxton N. Molecular evidence for the transovarial passage of Babesia gibsoni in Haemaphysalis hystricis (Acari: Ixodidae) ticks from Taiwan: a novel vector for canine babesiosis. Parasit Vectors 2018; 11: 134. https://doi.org/10.1186/ s13071-018-2722-y

6. Piranda EM, Faccini JL, Pinter A, Pacheco RC, Cançado PH, Labruna MB. Experimental infection of Rhipicephalus sanguineus ticks with the bacterium Rickettsia rickettsii, using experimentally infected dogs. Vector Borne Zoonotic Dis 2011; 11: 29-36. https://doi. org/10.1089/vbz.2009.0250

7. Dantas-Torres F, Martins TF, de Paiva-Cavalcanti M, Figueredo LA, Lima BS, Brandão-Filho SP. Transovarial passage of Leishmania infantum $\mathrm{kDNA}$ in artificially infected Rhipicephalus sanguineus. Exp Parasitol 2010; 125: 184-185. https://doi.org/10.1016/ j.exppara.2010.02.003

8. Horta MC, Pinter A, Schumaker TT, Labruna MB. Natural infection, transovarial transmission, and transstadial survival of Rickettsia bellii in the tick Ixodes loricatus (Acari: Ixodidae) from Brazil. Ann NY Acad Sci 2006; 1078: 285-290. https://doi.org/10.1196/ annals. 1374.053

9. Miller RJ, Davey RB, George JE. Modification of the food and agriculture organization larval packet test to measure amitraz-susceptibility against Ixodidae. J Med Entomol 2002; 39: 645-651. https://doi.org/10.1603/0022-2585-39.4.645

10. White WH, Plummer PR, Kemper CJ, Miller RJ, Davey RB, Kemp DH, Hughes S, Smith CK 2nd, Gutierrez JA. An in vitro larval 
immersion microassay for identifying and characterizing candidate acaricides. J Med Entomol 2004; 4: 1034-1042. https://doi. org/10.1603/0022-2585-41.6.1034

11. Mehlhorn H, Hansen O, Mencke N. Comparative study on the effects of three insecticides (fipronil, imidacloprid, selamectin) on developmental stages of the cat flea (Ctenocephalides felis Bouche 1835): a light and electron microscopic analysis of in vivo and in vitro experiments. Parasitol Res 2001; 87: 198-207. https://doi. org/10.1007/PL00008575

12. Mehlhorn $H$, Schmahl G, Mencke $N$, Bach T. In vitro and in vivo studies on the effect of a combination containing 10\% imidacloprid and 50\% permethrin against Ixodes ricinus ticks. Parasitol Res 2003; 89: 323-325. https://doi.org/10.1007/s00436-002-0809-y

13. Beugnet F, Franc M. Insecticide and acaricide molecules and/or combinations to prevent pet infestation by ectoparasites. Trends Parasitol 2012; 28: 267-279. https://doi.org/10.1016/j.pt.2012.04.004

14. Mehlhorn H, Mencke N, Hansen O. Effects of imidacloprid on adult and larval stages of the flea Ctenocephalides felis after in vivo and in vitro application: a light-and electron-microscopy study. Parasitol Res 1999; 85: 625-637. https://doi.org/10.1007/s004360050607

15. Stanneck D, Rass J, Radeloff I, Kruedewagen E, Le Sueur C, Hellmann K, Krieger K. Evaluation of the long-term efficacy and safety of an imidacloprid $10 \%$ /flumethrin $4.5 \%$ polymer matrix collar $\left(\right.$ Seresto $\left.{ }^{\circledR}\right)$ in dogs and cats naturally infested with fleas and/or ticks in multicentre clinical field studies in Europe. Parasit Vectors 2012; 5: 66. https://doi.org/10.1186/1756-3305-5-66

16. Yamaguti N, Tipton V, Keegan H, Toshioka S. Ticks of Japan, Korea, and the Ryukyu Islands: Brigham Young Univ Sci Bull Biol Ser 1971; 45: 1-226. https://scholarsarchive.byu.edu/byuscib/ vol15/iss1/1

17. Marchiondo A, Holdsworth P, Fourie L, Rugg D, Hellmann K, Snyder D, et al. World Association for the Advancement of Veterinary Parasitology (WAAVP): guidelines for evaluating the efficacy of parasiticides for the treatment, prevention and control of flea and tick infestations on dogs and cats. Parasit Vectors 2013; 194: 84-97. https://doi.org/10.1016/j.vetpar.2013.02.003

18. Hagimori I, Machida H, Goi R, Mencke N. Efficacy of imidacloprid/permethrin and fipronil/(S)-methoprene combinations against Haemaphysalis longicornis ticks evaluated under in vitro and in vivo conditions. Parasitol Res 2005; 97 (suppl): 120-126. https://doi.org/10.1007/s00436-005-1455-y

19. Borges L, Ferri P, Silva W, Silva W, Silva J. In vitro efficacy of extracts of Melia azedarach against the tick Boophilus microplus. Med Vet Entomol 2003; 17: 228-231. https://doi.org/10.1046/j.13652915.2003.00426.x

20. Hoogstraal H, Roberts FHS, Kohls GM, Tipton VJ. Review of Haemaphysalis (Kaiseriana) longicornis Neumann (resurrected) of Australia, New Zealand, New Caledonia, Fiji, Japan, Korea, and northeastern China and USSR, and its parthenogenetic and bisexual populations (Ixodoidea, Ixodidae). J Parasitol 1968; 54: 1197-1213. https://doi.org/10.2307/3276992

21. Roberts FHS. Australian Ticks. Melbourne, Australia. Common- wealth Scientific and Industrial Research Organization. 1970. https://www.cabdirect.org/cabdirect/abstract/19711001240

22. Rainey T, Occi JL, Robbins RG, Egizi A. Discovery of Haemaphysalis longicornis (Ixodida: Ixodidae) Parasitizing a Sheep in New Jersey, United States. J Med Entomol 2018; 55: 757-759. https:// doi.org/10.1093/jme/tjy006

23. Heath AC, Palma RL, Cane RP, Hardwick S. Checklist of New Zealand ticks (Acari: Ixodidae, Argasidae). Zootaxa 2011; 2995: 55-63. https://doi.org/10.5281/zenodo.203775

24. Luo LM, Zhao L, Wen HL, Zhang ZT, Liu JW, Fang LZ, Xue ZF, Ma DQ, Zhang XS, Ding SJ, Lei XY, Yu XJ. Haemaphysalis longicornis ticks as reservoir and vector of severe fever with thrombocytopenia syndrome virus in China. Emerg Infect Dis 2015; 21: 1770-1776. https://doi.org/10.3201/eid2110.150126

25. Heath A. Biology, ecology and distribution of the tick, Haemaphysalis longicornis Neumann (Acari: Ixodidae) in New Zealand. N Z Vet J 2016; 64: 10-20. https://doi.org/10.1080/00480169.20 15.1035769

26. Higuchi S, Simomura S, Yoshida H, Hoshi F, Kawamura S, Yasuda Y. Development of Babesia gibsoni in the gut epithelium of the tick, Haemaphysalis longicornis. J Vet Med Sci 1991; 53: 129-131. https://doi.org/10.1292/jvms.53.129

27. Murata T, Inoue $M$, Taura Y, Nakama S, Abe H, Fujisaki K. Detection of Hepatozoon canis oocyst from ticks collected from the infected dogs. J Vet Med Sci 1995; 57: 111-112. https://doi.org/10.1292/ jvms.57.111

28. Lee JH, Park HS, Jung KD, Jang WJ, Koh SE, Kang SS, Lee IY, Lee WJ, Kim BJ, Kook YH, Park KH, Lee SH. Identification of the spotted fever group rickettsiae detected from Haemaphysalis longicornis in Korea. Microbiol Immunol 2003; 47: 301-304. https:// doi.org/10.1111/j.1348-0421.2003.tb03399.x

29. Sun J, Liu Q, Lu L, Ding G, Guo J, Fu G, Zhang J, Meng F, Wu H, Song X, Ren D, Li D, Guo Y, Wang J, Li G, Liu J, Lin H. Coinfection with four genera of bacteria (Borrelia, Bartonella, Anaplasma, and Ehrlichia) in Haemaphysalis longicornis and Ixodes sinensis ticks from China. Vector Borne Zoonotic Dis 2008; 8: 791-796. https://doi.org/10.1089/vbz.2008.0005

30. Fourie JJ, Horak IG, De Vos C, Deuster K, Schunack B. Comparative speed of kill, repellent (anti-feeding) and acaricidal efficacy of an Imidacloprid/Flumethrin Collar (Seresto ${ }^{\circledR}$ ) and a Fipronil/ (S)-Methoprene/Eprinomectin/Praziquantel Spot-on (Broadline ${ }^{\circledR}$ ) against Ixodes ricinus (Linné, 1758) on cats. Parasitol Res 2015; 114: 109-116. https://doi.org/10.1007/s00436-015-4517-9

31. Gomez SA, Picado A. Systemic insecticides used in dogs: potential candidates for phlebotomine vector control? Trop Med Int Health 2017; 22: 755-764. https://doi.org/10.1111/tmi.12870

32. Dobson P, Tinembart O, Fisch R, Junquera P. Efficacy of nitenpyram as a systemic flea adulticide in dogs and cats. Vet Rec 2000; 147: 709-713. https://doi.org/10.1136/vr.147.25.709

33. Rust MK. Advances in the control of Ctenocephalides felis (cat flea) on cats and dogs. Trends Parasitol 2005; 21: 232-236. https://doi. org/10.1016/j.pt.2005.03.010 
